\title{
El oso melero (Tamandua mexicana) en el Caribe colombiano: aportes sobre su ecología y amenazas
}

\author{
Cesar Rojano ${ }^{\text {a,1, }}$ Julio Chacón Pacheco ${ }^{\text {y }}$ Andrés Felipe Polo ${ }^{C}$ \\ A Proyecto de conservación de hormigueros de Colombia, Fundación Cunaguaro, Calle 20 \#28-06, Yopal, Casanare, Colombia. \\ E-mail: c.rojanob@gmail.com

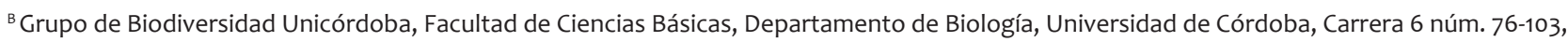 \\ Montería, Córdoba, Colombia. E-mail: jchacon_bio@hotmail.com \\ cFundación Universitaria San Martín, Antigua Vía Puerto Colombia (Cra. 51B) Km. 8, Barranquilla, Colombia. \\ E-mail: andrespollocc@hotmail.com \\ ${ }^{1}$ Autor para correspondencia
}

\begin{abstract}
Resumen El objetivo de este trabajo es aportar información sobre la ecología y amenazas del oso melero (Tamandua mexicana) en la región Caribe colombiana. Para estimar la densidad poblacional se realizaron 10 transectos lineales en Pedraza, Magdalena. De igual forma, se acumuló un recorrido de 1.356 km en la Vía Parque Nacional Natural Isla Salamanca, Magdalena, para determinar la frecuencia de atropellamientos en las vías. Por último, se recopilaron relatos de conflictos con perros domésticos en cuatro departamentos del Caribe. La densidad poblacional de osos meleros en Pedraza fue de 0,81 ind $/ \mathrm{km}^{2}$ y la tasa de encuentro de 2,43 ind / $100 \mathrm{~km}$. La tasa de atropellamientos durante el periodo de estudio en Vía Parque Isla Salamanca fue de 0,006 ind $/ \mathrm{km}$, mientras que la frecuencia fue 0,6 ind/día. Se recopilaron cuatro sucesos de conflicto con perros, uno de ellos causante de la muerte del ejemplar silvestre. Estos datos podrán utilizarse para diseñar futuros planes de conservación, atendiendo los procesos de transformación de los ecosistemas naturales que sufre la región Caribe colombiana.
\end{abstract}

Palabras clave: atropellamientos, Colombia, densidad, Vermilingua, Xenarthra

The northern tamandua (Tamandua mexicana) in the Colombian Caribbean: contributions on its ecology and threats

Abstract The aim of this study was to provide information about the ecology and threats of the northern tamandua (Tamandua mexicana) in the Caribbean region of Colombia. Linear transects were performed to estimate the population density. Similarly, the frequency of road collisions was determined by monitoring a total of 1,356 km of the Vía Parque Nacional Natural Isla Salamanca road, Magdalena. Finally, reports of conflicts with domestic dogs were collected in four departments of the Caribbean region. The population density of the northern tamandua in the study area was $0.81 \mathrm{ind} / \mathrm{km}^{2}$ and the encounter rate 2.43 ind $/ 100$ $\mathrm{km}$. The road kill rate in Isla Salamanca was estimated at $0.006 \mathrm{ind} / \mathrm{km}$ and a frequency of $0.6 \mathrm{ind} /$ day. Four incidents with dogs were reported; one of them resulted in the death of a tamandua. These results may be used to design future conservation plans, taking into account natural ecosystem transformation processes in the Caribbean region of Colombia.

Keywords: Colombia, density, road kills, Vermilingua, Xenarthra 


\section{INTRODUCCIÓN}

El oso melero Tamandua mexicana (Saussure, 1860) es un hormiguero de tamaño mediano, de hábitos principalmente arborícolas y dieta insectívora (Navarrete \& Ortega, 2011). Su distribución se extiende desde el sur de México hasta el noroeste de Perú y el noroeste de Venezuela. Se encuentra desde el nivel del mar hasta $2.500 \mathrm{msnm}$, aunque la mayoría de los avistamientos se ha registrado en zonas por debajo de 1.000 msnm (Eisenberg, 1989; Tirira, 2007; Superina et al., 2010).

Se puede encontrar en la zona tropical y los bosques secos y húmedos subtropicales, en manglares y pastizales con algunos árboles (Ortega-Reyes et al., 2014) y en bosques secundarios y hábitats perturbados (Navarrete \& Ortega, 2011). Ha sido reportado para la región Andina, en los departamentos de Antioquia, Boyacá, Caldas, Cundinamarca, Quindío, Huila, Norte de Santander, Risaralda, Santander y Tolima; para la región Pacífica en Cauca, Chocó, Nariño y Valle del Cauca; y en el Caribe colombiano para los departamentos de Atlántico, Bolívar, Córdoba, Cesar, La Guajira, Magdalena y Sucre (Humanez-López \& Chacón-Pacheco, 2014; Alzate-Gaviria et al., 2016).

Sin embargo, es uno de los xenartros menos conocidos en el país (Rojano et al., 2014) y se desconoce en profundidad diferentes aspectos de su biología y sus principales amenazas. Aunque no se encuentra listada en el Libro Rojo de mamíferos, ni en los listados actuales de especies amenazadas de Colombia (Rodríguez-Mahecha et al., 2006; MADS, 2014), se reconoce como una de las especies de mamíferos más afectadas por el tráfico ilegal de fauna silvestre en la región Caribe colombiana (Humanez-López et al., 2015). Sumado a esto, existe la probabilidad de que $T$. mexicana esté siendo afectado en toda su área de distribución por diversos factores, como atropellamientos, quemas, conflictos con perros y transformación de hábitat (Superina et al., 2010). Por tal motivo se hace necesario contribuir al conocimiento sobre el estado actual de la especie en Colombia, para así poder tomar medidas de conservación para este mamífero. Por lo tanto, el objetivo de este trabajo fue aportar información sobre la ecología y amenazas del oso melero (T. mexicana) en la región Caribe colombiana.

\section{MATERIALES Y MÉTODOS}

\section{Área de estudio}

La región Caribe de Colombia cuenta con una extensión de $132.218 \mathrm{~km}^{2}$ que abarca desde el noreste del golfo de Urabá hasta la península de La Guajira, y desde las estribaciones de las cordilleras Occidental y Central hasta las playas del mar Caribe. En general es una región llana, atravesada por los ríos Magdalena, Cauca, San Jorge, Sinú, Ranchería y Ariguaní que forman cerca de la costa amplias ciénagas y lagunas. Está conformada por los departamentos de Atlántico,
Bolívar, Cesar, Córdoba, La Guajira, Magdalena, San Andrés, Providencia y Santa Catalina, y Sucre. Su relieve se contrarresta con la Sierra Nevada de Santa Marta, la formación montañosa litoral más alta del mundo, una extensa zona con gran diversidad climática (Vásquez \& Buitrago, 2011).

Desde el punto de vista ecológico, la región alberga un complejo mosaico de ecosistemas terrestres y acuáticos que es bastante heterogéneo debido a que presenta unos rasgos climáticos, hidrológicos y topográficos bien diferenciados. Los ambientes terrestres se catalogan por su cobertura vegetal predominante, la cual incluye bosque húmedo tropical, bosque montano, bosque premontano, bosque seco tropical, bosque bajo denso, pastizales y matorrales semidesérticos. Sin embargo, gran parte de estos ecosistemas naturales se ha transformado para el desarrollo de actividades agropecuarias, en su mayoría extensivas (Díaz-Merlano, 2014).

\section{Muestreos}

Los análisis de densidad, atropellamientos y ataques de perros se llevaron a cabo en las siguientes localidades de la región Caribe:

- El estimativo de densidad poblacional se desarrolló en la cabecera municipal de Pedraza, Magdalena $\left(10^{\circ} 11^{\prime} 18^{\prime \prime} \mathrm{N}, 74^{\circ} 54^{\prime} 59^{\prime \prime} \mathrm{W}\right.$; FIG. 1). El área correspondió a $71,78 \mathrm{~km}^{2}$, consistiendo básicamente en potreros arbolados para ganadería extensiva, con presencia de pastos introducidos, especies nativas y exóticas de árboles y arbustos. La temperatura promedio en Pedraza es de $28^{\circ} \mathrm{C}$ y la humedad relativa de $70 \%$ (Padilla \& Almentero, 2012).

- La tasa de atropellamientos de T. mexicana se evaluó en el trayecto comprendido entre los kilómetros 3 y 48 de la vía que comunica a Barranquilla con Santa Marta, 45,2 km en una vía asfaltada de doble sentido y un ancho aproximado de $7 \mathrm{~m}\left(11^{\circ} 00^{\prime} \mathrm{N}\right.$, $74^{\circ} 40^{\prime}$ W; FIG. 2). La temperatura promedio es de 29 ${ }^{\circ} \mathrm{C}$, con una humedad relativa del $70 \%$. Los primeros $4,3 \mathrm{~km}$ se encuentran bordeados por un mosaico de parcelas donde se llevan a cabo actividades agropecuarias, como ganadería extensiva y cultivos de subsistencia. Los 40,9 km restantes comprenden la Vía Parque Nacional Natural Isla Salamanca $\left(10^{\circ} 56^{\prime} \mathrm{N}\right.$, $74^{\circ} 27^{\prime} \mathrm{W}$ ), un conjunto de playones, ciénagas y bosques que comprende un área total de 56.200 ha, pertenecientes al delta del estuario del río Magdalena en el departamento de Magdalena. Este trayecto está bordeado por una zona de bosque seco tropical, con presencia de bosques de manglares con predominancia de Rhizophora mangle (mangle rojo), Avicennia germinans (mangle salado), Laguncularia racemosa (mangle amarillo) y Conocarpus erecta (mangle zaragoza o bobo; Ulloa-Delgado et al., 1998; Moreno-Bejarano \& Álvarez-León, 2003). 


\section{Densidad poblacional}

Para la estimación de densidad poblacional se recorrieron diez transectos lineales de 4 a $8 \mathrm{~km}$ de largo, trazados aleatoriamente sobre potreros y vías al interior de sistemas agropecuarios en el área de estudio en el municipio de Pedraza. Entre abril y agosto de 2012 cada transecto fue recorrido una vez al mes. Las observaciones se realizaron al amanecer entre las 6:00 y 8:00 hs y al atardecer entre las 16:00 y 18:00 hs. Cada transecto fue recorrido de ida y vuelta (réplica) a una velocidad de 2 a $3 \mathrm{~km} / \mathrm{h}$, dejando un lapso de tiempo de $30 \mathrm{~min}$ entre recorridos. De cada observación se consignaron datos como fecha, hora, distancia

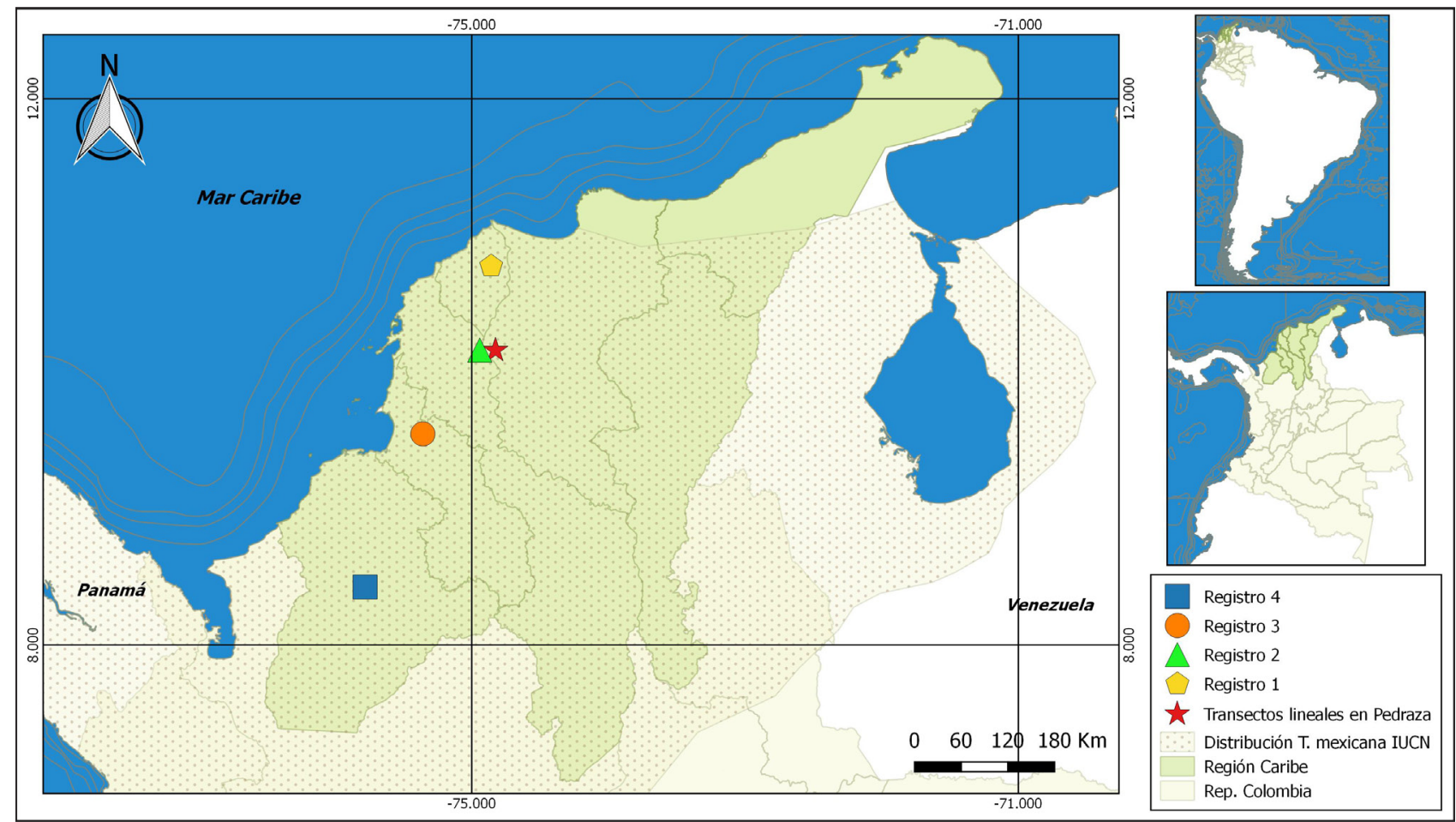

FIGURA 1. Localidad de transectos lineales en Pedraza, Magdalena y registros de ataques de perros a osos meleros (Tamandua mexicana) en el Caribe colombiano.

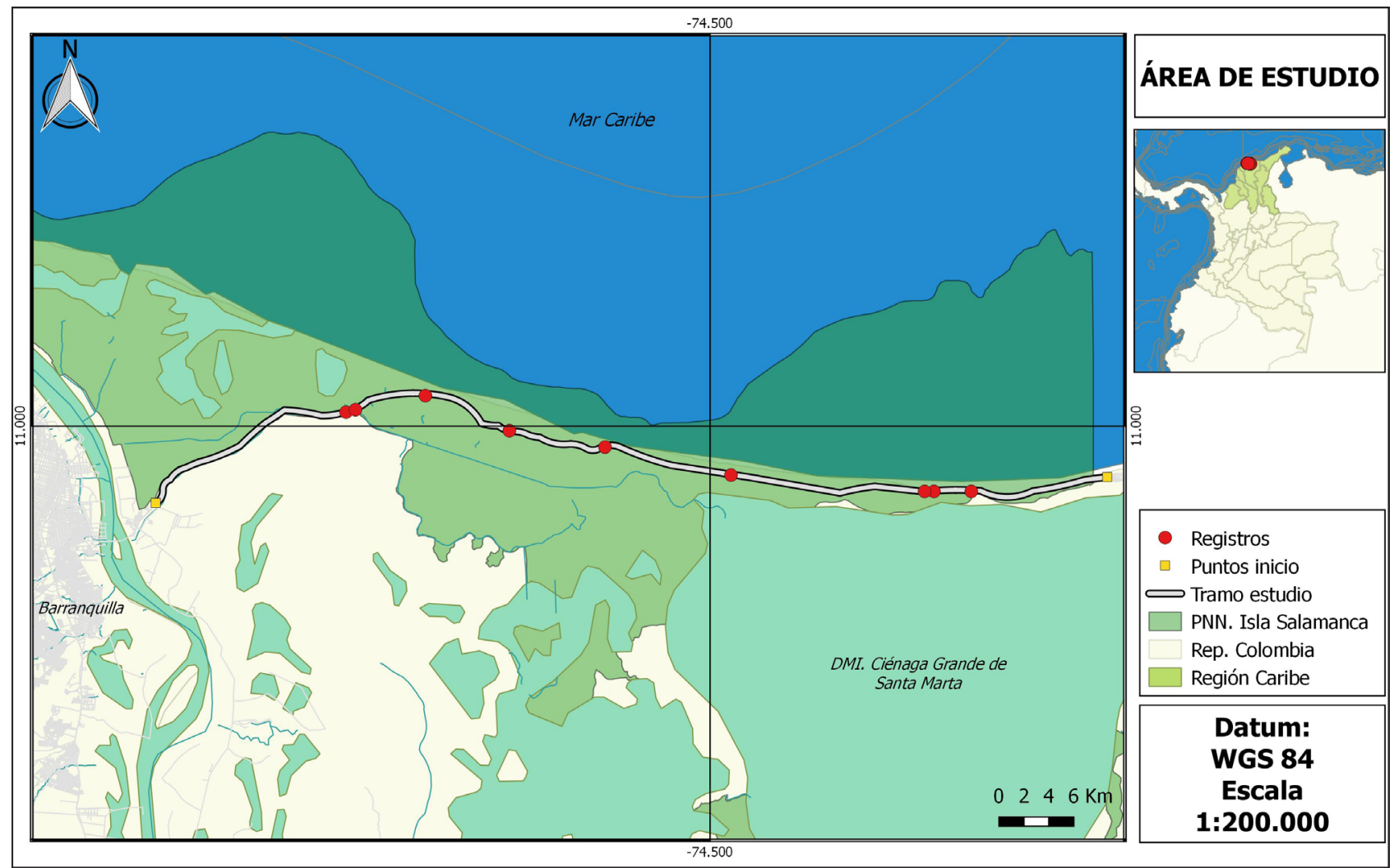

FIGURA 2. Registros de atropellamientos de Tamandua mexicana en el Caribe colombiano. 
aproximada del observador al animal, comportamiento y ubicación del ejemplar (en árboles o en el suelo). Se registraron las coordenadas geográficas sobre el transecto en dirección perpendicular al animal con un GPS (Garmin eTrex 20, Lenexa, Estados Unidos).

Debido al bajo número de observaciones registradas, se utilizó el método de transectos lineales (Burnham et al., 1980) para la estimación de la densidad poblacional. El cálculo se obtuvo a partir de la siguiente fórmula matemática:

$$
D=\frac{N}{(2 \times E S W \times L)}
$$

Donde $\mathrm{D}=$ densidad; $\mathrm{N}$ = número de animales avistados; ESW = ancho efectivo visual del área muestreada (en $\mathrm{km}$ ) y $\mathrm{L}=\mathrm{km}$ totales recorridos. Con base en los registros obtenidos en campo, se estableció un ancho promedio de transecto de $15 \mathrm{~m}$.

\section{Amenazas}

Atropellamiento en carreteras. Este estudio se desarrolló en el mes de julio de 2014, época de lluvias en el Caribe colombiano. El periodo de estudio fue de 15 días durante los cuales el tramo de interés fue recorrido en horas de la mañana (6:00-8:00 hs) y posteriormente en horas de la tarde (16:00-18:00 hs) con el fin de determinar en qué periodo del día se presentaba el mayor número de atropellamientos. Los recorridos fueron llevados a cabo por dos observadores que se desplazaban en una motocicleta a una velocidad promedio de $20 \mathrm{~km} / \mathrm{h}$ (Hawbaker \& Radeloff, 2004; Seijas et al., 2013). De cada ejemplar atropellado se consignó su ubicación con un GPS para evitar reconteos de individuos. Para el análisis de los datos se calculó la frecuencia de atropellamientos por día; de igual forma se obtuvo la tasa de atropellamientos de T. mexicana por kilómetros.

Conflicto con perros domésticos. Se recopilaron registros de ataques de perros domésticos a individuos de T. mexicana entre los años 2012 y 2013, correspondientes a observaciones directas de los investigadores en algunas zonas de la Región Caribe de Colombia. Estas observaciones fueron producto del trabajo en campo durante los años mencionados y del ejercicio de atención médico veterinaria de los ejemplares silvestres prestada por uno de los investigadores a las autoridades ambientales.

\section{RESULTADOS}

\section{Densidad poblacional}

En total se recorrieron $576 \mathrm{~km}$, avistando 14 ejemplares de T. mexicana. La densidad poblacional en el área de estudio fue de $0,81 \mathrm{ind} / \mathrm{km}^{2}$. La tasa de encuentro fue de 2,43 ind $/ 100 \mathrm{~km}$. El 100\% de los ejemplares observados se encontraban en árboles. Ocho de los individuos observados descansaban dentro de troncos o nidos de termitas abandonados. Los seis restantes se encontraban forrajeando.

\section{Amenazas}

Atropellamientos en vías. En total se recorrieron $1.356 \mathrm{~km}$ de vías, registrando nueve individuos de $T$. mexicana atropellados durante los 15 días de muestreo (FIG. 2). La tasa de atropellamientos durante el periodo de estudio fue de 0,006 ind $/ \mathrm{km}$, mientras que la frecuencia fue 0,6 ind/ día. El 100\% de los individuos muertos se registró en los recorridos de la mañana, lo que sugiere que todos los atropellamientos se presentaron en horas de la noche.

Conflictos con perros domésticos. A continuación se presentan los relatos de registros de ataques en diferentes localidades del Caribe colombiano (FIG. 1).

\section{- Registro \#1}

El 14 de junio de 2012 se registró un individuo macho adulto de T. mexicana en un cultivo de maíz en la zona rural de Pedraza, Magdalena $\left(10^{\circ} 12^{\prime} 16^{\prime \prime} \mathrm{N}\right.$, $\left.74^{\circ} 54^{\prime} 48^{\prime \prime} \mathrm{W}\right)$. Se recibió la llamada del propietario del predio, manifestando que sus perros estaban atacando un oso melero que había bajado de un árbol en horas de la tarde. Al llegar al lugar el ejemplar se encontraba muerto, con lesiones en distintas partes del cuerpo producto de las mordeduras. Uno de los peros presentaba algunas lesiones cutáneas producto de las garras del T. mexicana.

- Registro \#2

Se presentó el día 6 de febrero de 2013, en zona rural del municipio de Polonuevo, Atlántico $\left(10^{\circ} 46^{\prime} 53^{\prime \prime} \mathrm{N}, 74^{\circ} 50^{\prime} 33^{\prime \prime} \mathrm{W}\right)$. El ejemplar hembra juvenil de T. mexicana fue atacado en horas de la madrugada por dos perros de vigilancia de una finca ganadera de la zona. Posterior a esto fue rescatado por el personal de seguridad y entregado a la Corporación Autónoma Regional del Atlántico, la cual lo remitió a una clínica veterinaria de la ciudad de Barranquilla donde fue evaluado. Se encontraron lesiones en cabeza y miembros anteriores que se trataron para posteriormente liberar al animal en un Ecoparque del departamento del Atlántico.

\section{- Registro \#3}

El 26 de abril de 2013 se registró en zona periurbana del municipio de Montería, Córdoba $\left(8^{\circ} 46^{\prime} 6^{\prime \prime} \mathrm{N}\right.$, $\left.75^{\circ} 51^{\prime} 29^{\prime \prime} \mathrm{W}\right)$, un individuo hembra adulta de T. mexicana que fue atacado por perros domésticos. Este ejemplar se encontraba forrajeando en un potrero utilizado para ganadería cuando fue agredido por algunos perros de la zona, siendo necesaria la intervención de la comunidad. Fue capturado y transportado a la ciudad de Montería, para posteriormente ser reubicado por la Corporación Autónoma Regional 
de los Valles del Sinú y San Jorge en otra zona del departamento. Durante la inspección clínica del animal sólo se encontraron pequeñas lesiones cutáneas focalizadas.

\section{- Registro \#4}

En septiembre de 2013 se observó en la zona rural del municipio de Colosó, Sucre ( $\left.9^{\circ} 31^{\prime} 19^{\prime \prime} \mathrm{N}, 75^{\circ} 21^{\prime} 53^{\prime \prime} \mathrm{W}\right)$, un individuo juvenil de T. mexicana que presentaba lesiones cutáneas compatibles con mordeduras de perros domésticos. El ejemplar se encontraba en una zona boscosa, colindante con algunas pequeñas fincas con cultivos de maíz y potreros destinados a ganadería extensiva, cuyos propietarios acostumbran tener perros destinados a la cacería de especies silvestres. La mayoría de lesiones se observaron en miembros anteriores y posteriores, así como en cuello y cola (FIG. 3). Sobre este ejemplar no se realizó ningún tipo de intervención posterior a la observación.

\section{Discusión}

Para Colombia no existen estimaciones previas de densidad de T. mexicana, e inclusive en toda el área de distribución de la especie se reportan pocos datos sobre este parámetro poblacional. Los valores de densidad poblacional publicados que se basaron en el método de transectos lineales en su mayoría son superiores a los obtenidos en potreros de Pedraza. En zonas boscosas de Barro Colorado, Panamá, se registraron densidades desde $1,4 \mathrm{ind} / \mathrm{km}^{2}$ hasta 5,7 ind $/ \mathrm{km}^{2}$ (Wright et al., 1994, 2000; Timock \& Vaughan, 2002). De igual forma, en el bosque húmedo de tierras bajas en Costa Rica se reportaron 5,7 ind $/ \mathrm{km}^{2}$ (Van Hulle \& Vaughan, 2009).

Diversos aspectos pueden influir en los valores de densidad de mamíferos. Este parámetro puede variar de acuerdo con la calidad y estado de conservación del hábitat (Eisenberg et al., 1979) y al área biogeográfica donde se encuentre (Peters \& Raelson, 1984). Se espera que sea inferior en hábitats menos productivos (Glanz, 1982). Los datos obtenidos en áreas degradadas de Pedraza en comparación con zonas boscosas indican que, si bien la especie podría tolerar actividades humanas, su densidad podría estar siendo afectada por la transformación del hábitat.

Las particularidades de cada individuo también pueden influir en los valores de densidad. Algunos autores proponen que los requerimientos alimenticios (Robinson \& Redford, 1986) y la capacidad de algunos individuos de T. mexicana de tener periodos de actividad diurnos, nocturnos o mixtos hacen que sus densidades sean difíciles de medir (Montgomery \& Lubin, 1977). Inclusive las metodologías usadas para realizar los censos (p. ej. transectos lineales, fototrampeo) pueden influir sobre los valores estimados (Van Hulle \& Vaughan, 2009; Desbiez \& Medri, 2010). Existe la probabilidad que algunos de estos factores hayan interferido con la precisión de las densidades poblacionales en Pedraza. Sin embargo, se requiere recopilar mayor información al respecto en el área de estudio.

En Colombia existen diversos reportes sobre el atropellamiento de T. mexicana, siendo mencionado,

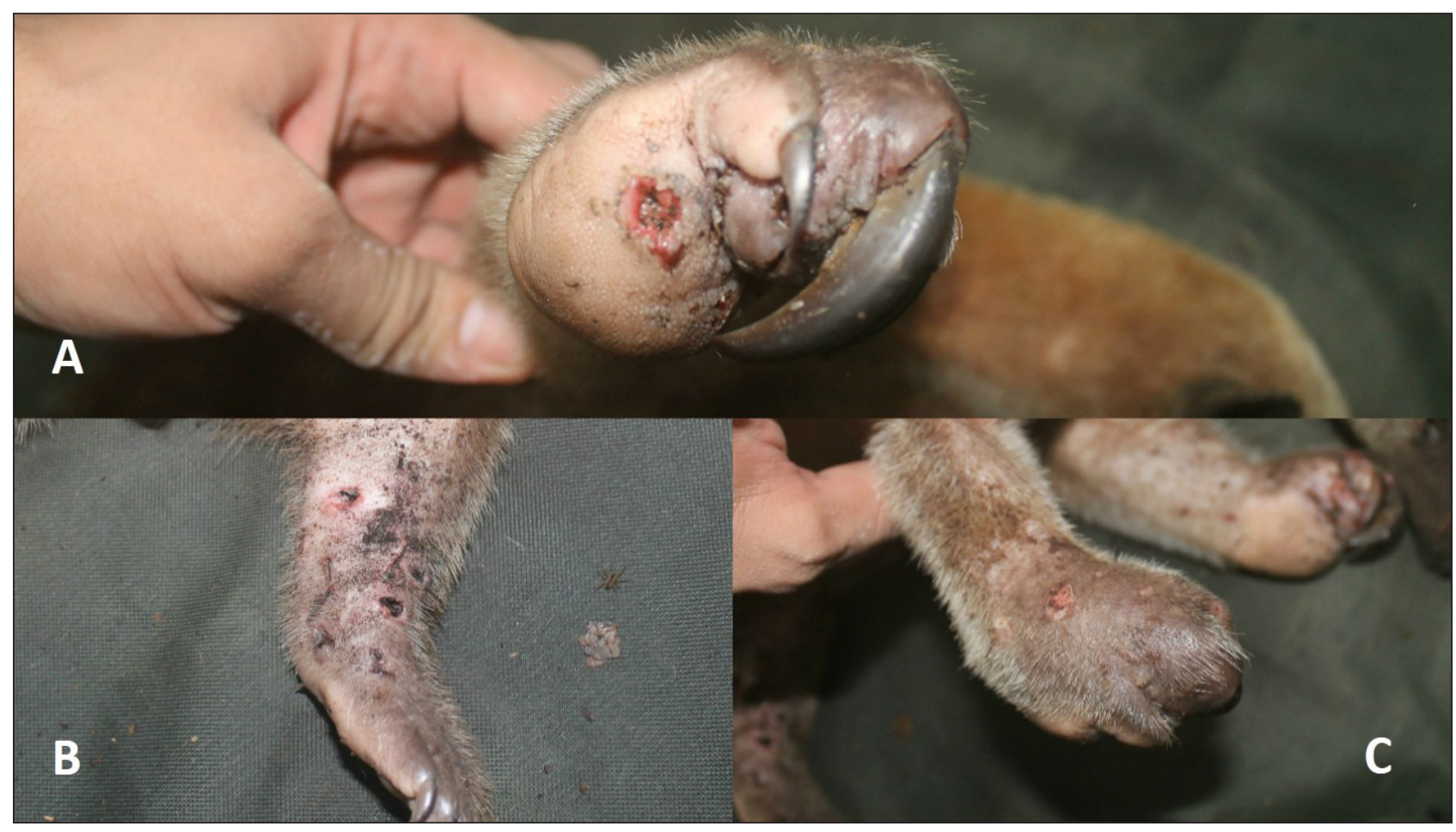

FIGURA 3. Lesiones cutáneas encontradas en un ejemplar juvenil de Tamandua mexicana en el Caribe colombiano, probablemente causadas por perros de la zona. A. Lesión compatible con mordedura en miembro anterior derecho, cara medial. B. Lesión en miembro posterior izquierdo, cara medial. C. Lesión en miembro anterior derecho, cara lateral. 
inclusive, como uno de los mamíferos más atropellados en algunos tramos viales del país (Argotte \& Monsalvo, 2002; Payán et al., 2013). En cuanto al Caribe colombiano, el oso melero ha sido reportado como una especie vulnerable al atropellamiento en seis de sus ocho departamentos (Sánchez-Páez et al., 2004; De la Ossa-Nadjar \& De la Ossa, 2013; Payán et al., 2013; Viloria-Rivas \& Chacón, 2014; De la Ossa \& Galván-Guevara, 2015; Monroy et al., 2015), lo que indica que es una problemática generalizada.

La tasa de atropellamiento de T. mexicana encontrada en este estudio fue superior a la reportada por Argotte \& Monsalvo (2002). Estos autores estimaron en Vía Parque Isla Salamanca una tasa de 0,0044 ind/ $\mathrm{km}$, registrando una mayor incidencia de muertes durante los meses de sequía. Por otro lado, en la Ruta del Sol, entre Santander y Magdalena, se reporta una tasa superior, de 0,0334 ind/km (Payán et al., 2013). Los resultados de estos trabajos evidencian que en el Caribe colombiano el oso melero está siendo afectado por los atropellamientos tanto en algunas áreas protegidas como por fuera de ellas. Sin embargo, el impacto real de esta amenaza sobre sus poblaciones aún se desconoce.

Diversos factores han sido considerados para explicar las tasas de atropellamiento de T. mexicana. Algunos autores afirman que los movimientos lentos y torpes de este animal sobre terreno lo hacen una especie muy vulnerable al atropello (Argotte \& Monsalvo, 2002; Carvajal-Alfaro \& Quesada, 2013). De igual forma, la dieta mirmecófaga podría jugar un papel importante dado que las carreteras favorecen potencialmente el establecimiento de colonias de hormigas en los bordes de las vías (Waide, 1991; De la Ossa \& Galván-Guevara, 2015).

El flujo de vehículos también puede ser un factor predisponente (Carvajal-Alfaro \& Quesada, 2013). La carretera que atraviesa el área protegida de Isla Salamanca presenta un alto valor de tránsito promedio diario (TPD), con 6.300 vehículos por día y picos de hasta 9.000 automóviles en temporada alta, a velocidades máximas de $90 \mathrm{~km} / \mathrm{h}$ (Mintransporte, 2006). El modelo de Tránsito Promedio Diario (TPD) (Seiler, 2003) afirma que vías con más de 4.000 vehículos por día pueden causar altas mortalidades de fauna, e incluso convertirse en una barrera permanente. Es probable que los efectos propuestos por el modelo TPD se estén presentando en Isla Salamanca. Dado que la especie es un objeto de conservación de esta área protegida, se requerirán grandes esfuerzos de monitoreo y medidas de mitigación del impacto de las vías sobre este hormiguero.

Se considera que las especies de hormigueros se ven afectadas por la presencia de fauna doméstica, que van desde los ataques hasta transmisión de enfermedades infecciosas (Koster, 2008; Miranda et al., 2015). Tradicionalmente, los perros domésticos son utilizados en la cacería de fauna silvestre, y aunque los hormigueros no constituyen una parte importante de la dieta de comunidades locales del Caribe, los encuentros entre perros y hormigueros son relativamente frecuentes (Rodriguez-Mahecha et al., 2006; Tirira, 2007; Koster, 2008; Koster \& Noss, 2014). Esto hace que los hormigueros queden expuestos a sufrir lesiones considerables e inclusive la muerte (Koster, 2008; Humanez \& Chacón-Pacheco, 2013), tal como se reporta en este estudio.

Adicional a esto, se ha reportado la presencia de perros ferales en zonas con abundante vida silvestre, lo cual puede generar aún más ataques a los hormigueros (Silva, 2015), causando disminuciones poblacionales que a largo plazo pueden generar aislamiento de las poblaciones, como se ha reportado para Myrmecophaga tridactyla (Lacerda et al., 2009).

Es probable que en toda el área de distribución de T. mexicana en el Caribe colombiano existan muy pocos fragmentos de bosque sin presencia de perros, ya sean domésticos o ferales. Es por esto que las medidas de conservación de esta especie a futuro deberán considerar nuevas áreas protegidas con poblaciones controladas de perros.

\section{AGRADECIMIENTOS}

Los autores agradecen los aportes y colaboración de Danielle Brown, Carlos M. Ospina, Javier Racero Casarrubia, Miguel Bacca, Dave Wehdeking, Juan García Hernández, Vicky Mejía Mogollón y José Francisco Zapata. De igual forma, a Roberto Martínez, Donis González y Rodolfo Osorio por su colaboración durante el trabajo de campo.

\section{REFERENCIAS}

Alzate-Gaviria, M., J. F. González-Maya \& A. BoteroBotero. 2016. Distribución geográfica y estado de conocimiento de las especies del género Tamandua (Xenarthra: Myrmecophagidae) en Colombia. Edentata 17: 8-16. https://doi.org/ 10.2301/IUCN.CH.2016.Edentata-17-1.3.en

Argotte, D. \& J. Monsalvo. 2002. Incidencia de la carretera Barranquilla-Ciénaga, sobre la mortalidad de vertebrados y su relación con el medio ecológico, en la Vía Parque Isla de Salamanca, Magdalena, Colombia. Tesis de pregrado, Universidad del Atlántico, Barranquilla, Colombia. $80 \mathrm{pp}$.

Burnham, K. P., D. R. Anderson \& J. L. Laake. 1980. Estimation of density from line transect sampling of biological populations. Wildlife Monographs 72: 3-202.

Carvajal-Alfaro, V. \& F. D. Quesada. 2013. Atropello de mamíferos silvestres en la ruta de acceso al cantón de Liberia, Guanacaste, Costa Rica. Revista Ventana 7: 12-14. 
De la Ossa, J. \& S. Galván-Guevara. 2015. Registro de mortalidad de fauna silvestre por colisión vehicular en la carretera Toluviejo-ciénaga La Caimanera, Sucre, Colombia. Biota Colombiana 16: 67-77.

De la Ossa-Nadjar, O. \& J. De la Ossa. 2013. Fauna silvestre atropellada en dos vías principales que rodean los Montes de María, Sucre, Colombia. Revista Colombiana de Ciencia Animal 5: 158-164.

Desbiez, A. L. J. \& Í. M. Medri. 2010. Density and habitat use by giant anteaters (Myrmecophaga tridactyla) and southern tamanduas (Tamandua tetradactyla) in the Pantanal wetland, Brazil. Edentata 11: 4-10. https: / / doi.org/10.1896/020.011.0102

Díaz-Merlano, J. M. 2014. Región Caribe de Colombia. Banco de Occidente, Cali, Colombia. 205 pp.

Eisenberg, J. F. 1989. Mammals of the Neotropics. Volume 1. The northern Neotropics: Panama, Colombia, Venezuela, Guyana, Suriname, French Guiana. University of Chicago Press, Chicago. 550 pp.

Eisenberg, J. F., M. O'Connell \& P. V. August. 1979. Density, productivity, and distribution of mammals in two Venezuelan habitats. Vertebrate Ecology in the Northern Neotropics: 187-207.

Glanz, W. E. 1982. The terrestrial mammal fauna of Barro Colorado island: Censuses and longterm changes. Pp. 455-468 in: Ecology of a tropical forest (E. G. Leigh, A. S. Rand \& D. M. Windsor, eds.). Smithsonian Institution Press, Washington, D.C.

Hawbaker, T. J. \& V. C. Radeloff. 2004. Roads and landscape pattern in northern Wisconsin based on a comparison of four road data sources. Conservation Biology 18: 1233-1244. https:// doi.org/10.1111/j.1523-1739.2004.00231.x

Humanez, E. \& J. Chacón-Pacheco. 2013. Nuevo registro de Myrmecophaga tridactyla para el departamento de Córdoba, Colombia con anotaciones sobre comportamiento agonístico interespecífico. Revista Colombiana de Ciencia Animal 5: 422-426.

Humanez-López, E. \& J. Chacón-Pacheco. 2014. Taxonomía, identificación y distribución de las especies del suborden Vermilingua en Colombia. Pp. 18-31 in: Manual de rehabilitación de hormigueros de Colombia (C. Rojano, L. Miranda \& R. Avila, eds.). Fundación Cunaguaro, Geopark Colombia S.A.S., Yopal, Colombia.

Humanez-López, E., J. Chacón-Pacheco \& T. Plese. 2015. Áreas de extracción de xenartros en el Caribe colombiano. Edentata 16: 65-68.

Koster, J. M. 2008. Hunting with dogs in Nicaragua: an optimal foraging approach. Current
Anthropology 49: 935-944. https://doi. org / $10.1086 / 592021$

Koster, J. \& A. Noss. 2014. Hunting dogs and the extraction of wildlife as a resource. Pp. 265-285 in: Free-ranging dogs and wildlife conservation (M. E. Gompper, ed.). Oxford University Press, Oxford.

Lacerda, A. C., W. Tomas \& J. Marinho-Filho. 2009. Domestic dogs as an edge effect in the Brasília National Park, Brazil: interactions with native mammals. Animal Conservation 12: 477-487. https: / / doi.org/10.1111/j.1469-1795.2009.00277.x

MADS - Ministerio de Ambiente y Desarrollo Sostenible. 2014. Resolución 0192 de 10 de febrero de 2014, República de Colombia. <https: / / www.minambiente.gov.co/images / normativa / resoluciones / 2014/res_0192_2014.pdf $>$. Consultada 26 de julio de 2015.

Mintransporte - Ministerio de Transporte de Colombia. 2006. Estudio que sirva de base para la regulación de precios del servicio público de transporte carretero de carga e intermunicipal de pasajeros. Tercer informe. Econometría Consultores, Ministerio de Transporte de Colombia, Bogotá D.C. 115 pp.

Miranda, F. R., M. Superina, F. Vinci, V. Hashimoto, J. C. Freitas \& E. R. Matushima. 2015. Serosurvey of Leptospira interrogans, Brucella abortus and Chlamydophila abortus infection in free-ranging giant anteaters (Myrmecophaga tridactyla) from Brazil. Pesquisa Veterinária Brasileira 35: 462-465. https:// doi.org/10.1590/ S0100-736X2015000500013

Monroy, M. C., A. De La Ossa-Lacayo \& J. De La Ossa. 2015. Tasa de atropellamiento de fauna silvestre en la vía San Onofre-María La Baja, Caribe colombiano. Revista de la Asociación Colombiana de Ciencias Biológicas 27: 88-95.

Montgomery, G. \& Y. Lubin. 1977. Prey influences on movements of Neotropical anteaters. Pp. 103-131 in Proceedings of the 1975 predator symposium (R. L. Phillips \& C. J. Jonkel, eds.), Missoula, MT.

Moreno-Bejarano, L. M. \& R. Álvarez-León. 2003. Fauna asociada a los manglares y otros humedales en el delta-estuario del río Magdalena, Colombia. Revista de la Academia Colombiana de Ciencias Exactas, Físicas y Naturales 27: 517-534.

Navarrete, D. \& J. Ortega. 2011. Tamandua mexicana (Pilosa: Myrmecophagidae). Mammalian Species 43: 56-63. https://doi. org / $10.1644 / 874.1$

Ortega-Reyes, J., D. G. Tirira, M. Arteaga \& F. Miranda. 2014. Tamandua mexicana. The IUCN Red List of Threatened Species 2014. <http:// 
dx.doi.org / 10.2305 / IUCN.UK.2014-1.RLTS. T21349A47442649.en.> Consultada 26 de junio de 2016.

Padilla, H. \& E. Almentero. 2012. Plan de manejo ambiental del municipio de Pedraza, Magdalena, Colombia. Informe final. Fundación Natureza, Pedraza, Colombia. 180 pp.

Payán, E., C. Soto, A. Díaz-Pulido, A. Benítez \& A. Hernández. 2013. Wildlife road crossing and mortality: lessons for wildlife friendly road design in Colombia. Pp. 1-16 in International conference on ecology and transportation, Scottsdale, Arizona.

Peters, R. H. \& J. V. Raelson. 1984. Relations between individual size and mammalian population density. The American Naturalist 124: 498-517. https: / / doi.org/10.1086/284290

Robinson, J. G. \& K. H. Redford. 1986. Body size, diet, and population density of Neotropical forest mammals. American Naturalist 128: 665680. https:/ / doi.org/10.1086/284596

Ramírez-Chaves, H. E. \& W. A. Pérez. 2010. Mamíferos (Mammalia: Theria) del departamento del Cauca, Colombia. Biota Colombiana 11: 141-171.

Rodríguez-Mahecha, J. V., M. Alberico, F. Trujillo \& J. Jorgenson. 2006. Libro Rojo de los mamíferos de Colombia. Conservación Internacional Colombia, Bogotá, Colombia. 433 pp.

Rojano, C., L. Miranda \& R. Ávila. 2014. Manual de rehabilitación de hormigueros de Colombia. Fundación Cunaguaro, Geopark Colombia S.A.S., Yopal, Colombia. 155 pp.

Sánchez-Páez, H., G. A. Ulloa-Delgado \& H. A. Tavera-Escobar. 2004. Manejo integral de los manglares por comunidades locales del Caribe de Colombia. Proyecto PD 60/01 REV. 1. Manejo sostenible y restauración de los manglares por comunidades locales del Caribe de Colombia. MAVDT, Dirección de Ecosistemas, CONIF, OIMT, Bogotá D.C. 335 pp.

Seijas, A. E., A. Araujo-Quintero \& N. Velásquez. 2013. Mortalidad de vertebrados en la carretera Guanare-Guanarito, estado Portuguesa, Venezuela. Revista de Biología Tropical 61: 1-18. https: / / doi.org/10.15517/ rbt.v61i4.12803

Seiler, A. 2003. Ecological effects of roads - a review. Introductory Research Essay 9. Department of Conservation Biology, Swedish University of Agricultural Sciences, Uppsala, Sweden. 40 pp.

Silva, E. A. 2012. Domestic dogs as invasive species: from local to global impacts. Tesis Doctoral, University of Florida, Gainesville. 180 pp.
Superina, M., F. R. Miranda \& A. M. Abba. 2010. The 2010 anteater Red List assessment. Edentata 11: 96-114. https:/ / doi.org/10.5537/020.011.0201

Timock, J. \& C. Vaughan. 2002. A census of mammal populations in Punta Leona private wildlife refuge, Costa Rica. Revista de Biología Tropical 50: 1169-1180.

Tirira, D. 2007. Guía de campo de los mamíferos del Ecuador. Publicación especial sobre los mamíferos del Ecuador 6. Ediciones Murciélago Blanco, Quito. 576 pp.

Ulloa-Delgado, G., G. S. Páez, H. G. Torres, W. P. Renjifo, J. R. Cruz \& H. A. León. 1998. Conservación y uso sostenible de los manglares del Caribe colombiano. Ministerio del Medio Ambiente, Asociación Colombiana de Reforestadores, OIMT, Proyecto Conservación y Manejo para el Desarrollo de los Manglares en Colombia, Bogotá D.C. 222 pp.

Van Hulle, M. \& C. Vaughan. 2009. The effect of human development on mammal populations of the Punta Leona private wildlife refuge, Costa Rica. Revista de Biología Tropical 57: 441-449.

Vásquez, A. \& A. Buitrago. 2011. El gran libro de los páramos. Instituto de Investigación de Recursos Biológicos Alexander von Humboldt, Proyecto Páramo Andino, Bogotá D.C. 208 pp.

Viloria-Rivas, J. \& J. Chacón. 2014. Impactos de las carreteras sobre los mamíferos silvestres: una realidad en el departamento de Córdoba, Colombia. Revista de la Asociación Colombiana de Ciencias Biológicas 26: 76.

Waide, R. B. 1991. Summary of the response of animal populations to hurricanes in the Caribbean. Biotropica 23: 508-512. https: / / doi. org $/ 10.2307 / 2388273$

Wright, S. J., M. E. Gompper \& B. De Leon. 1994. Are large predators keystone species in Neotropical forests? The evidence from Barro Colorado Island. Oikos 71: 279-294. https: / / doi. org / 10.2307/3546277

Wright, S. J., H. Zeballos, I. Domínguez, M. M. Gallardo, M. C. Moreno \& R. Ibáñez. 2000. Poachers alter mammal abundance, seed dispersal, and seed predation in a Neotropical forest. Conservation Biology 14: 227-239. https:/ / doi.org/10.1046/j.1523-1739.2000.98333.x

Recibido: 7 de septiembre de 2016; Aceptado: 4 de diciembre de 2016 